CASE REPORT

M.A. Mogensen

A.C. Lin

K.W. Chang

G.J. Berry

P.D. Barnes

N.J. Fischbein

\title{
Salivary Gland Anlage Tumor in a Neonate Presenting with Respiratory Distress: Radiographic and Pathologic Correlation
}

SUMMARY: We present a case of congenital salivary gland anlage tumor (SGAT) of the nasal septum in a 2-week-old infant who had difficulty breathing through her nose since birth. CT and MR imaging demonstrated a circumscribed mass within the nasal cavity that did not communicate with the intracranial compartment. Differential diagnosis and clinical significance of recognizing this rare lesion are reviewed.

$\mathbf{N}$ asal airway obstruction in the neonatal period is a potentially life-threatening condition that can be caused by a wide range of entities including congenital malformations, inflammatory conditions, hamartomas, and tumors. If a nasal mass is identified, then CT and MR imaging is often required to further characterize the mass and assess for intracranial involvement before intervention. Congenital salivary gland anlage tumor (SGAT) is a rare, benign lesion of the nasopharynx that presents with respiratory distress in the first days or weeks of life. Although this rare entity has been reported in the surgical and pediatric literature, its imaging features have not been reported in detail.

\section{Case Report}

A 2-week-old infant had trouble breathing through her nose since birth. The patient's pediatrician noted some bleeding when passing a catheter through the left naris, and the patient was referred to a pediatric otolaryngologist. A nasal endoscopy revealed a friable mass filling the left nasal cavity at the level of the middle turbinate. The right nasal cavity was narrowed because of a severe right septal deviation.

CT without contrast enhancement showed a soft tissue attenuation mass centered within the left nasal cavity with benign-appearing bony remodeling of the ethmoid labyrinth. A connection between the mass and the intracranial contents could not be excluded because of normal lack of ossification of the skull base at this age. An MR imaging examination was then performed and demonstrated a well-defined mass filling the posterior left nasal cavity that was T1 isointense and T2 iso-hyperintense to the brain parenchyma, with an intact skull base and no evidence of intracranial communication (Fig $1 A-C$ ).

The patient underwent CT-guided endoscopic resection of the mass, which was found to be pedicled to an approximately 4-mm area of the nasal septum. The choana and nasopharynx were clear.

The fragmented tissue measured $3.0 \times 2.3 \times 0.8 \mathrm{~cm}$ in aggregate. Microscopic examination revealed an overlying nonkeratinized squamous cell lining with foci of mucosal erosions. Nests of epithelial cells arranged as cords, and ductal and solid structures extended from the

Received August 25, 2008; accepted after revision September 12.

From the Department of Radiology (M.A.M., N.J.F.), Division of Neuroradiology, and Department of Pathology (G.J.B.), Stanford University Medical Center, Stanford, Calif; and Department of Otolaryngology (A.C.L., K.W.C.) and Division of Pediatric Otolaryngology and Radiology (P.D.B.), Stanford University, Lucile Packard Children's Hospital, Stanford, Calif.

Please address correspondence to Monique A. Mogensen, MD, Stanford University Medical Center, Department of Radiology, Division of Neuroradiology, 300 Pasteur Dr, Room S047, Stanford, CA 94305-5105; e-mail: moniquemogensen@stanford.edu

DOI 10.3174/ajnr.A1364

mucosal layer into an edematous submucosa. The ductlike structures contained foci of squamous differentiation surrounded by round and spindled stromal cells (Fig 2). Cellular pleomorphism and mitotic figures were absent. No neural, glial, or rhabdomyomatous differentiation was present. These findings were compatible with an SGAT.

\section{Discussion}

The term SGAT was first used in the literature by Dehner et al ${ }^{1}$ in 1994. They reported 9 cases of SGATs within the nasopharynx, all of which were midline and were attached to the posterior pharyngeal wall by a delicate pedicle. The tumors had a biphasic histologic pattern of squamous nests and ductlike structures along the periphery with solid, predominantly mesenchymal-appearing nodules centrally. Given the histologic similarity to the developing salivary gland, it was proposed that the SGAT is probably a hamartoma of minor salivary gland origin rather than a true neoplasm. Before this study, other similar lesions likely representing SGATs of the nasopharynx were discussed in the English-language literature, including a report in 1980 of a squamous cell proliferative le$\operatorname{sion}^{2}$ and a report in 1985 of a congenital pleomorphic adenoma. ${ }^{3}$

Congenital SGAT has a male predilection and is a rare cause of neonatal airway obstruction, with approximately 23 previous cases reported in the literature. ${ }^{4}$ The mass is midline and is typically attached to the posterior septum or the posterior nasopharyngeal wall by a thin pedicle. Excellent results have been obtained with simple excision, with no reported local recurrences. Two tumors were expelled during nasal manipulation (one during resuscitation and the other with nasal cannulation) ${ }^{1,5}$

Studies with CT and MR imaging are essential to evaluate anatomy, further define the characteristics and size of the mass, and assess for intracranial extension before surgical intervention. On CT examination, the homogeneous appearance of the mass with absence of bony destruction suggested a benign cause. Because of lack of ossification of the skull base, an MR imaging study was needed to assess for a nasoethmoidal encephalocele, which contains brain tissue of varied signal intensity on T2-weighted imaging. ${ }^{6}$ The intact skull base was clearly visualized on MR imaging. Although gadolinium contrast was not given in this case because of the absence of intravenous access, 1 previous case in the literature reported heterogeneous gadolinium enhancement in a SGAT. ${ }^{4}$ On the 

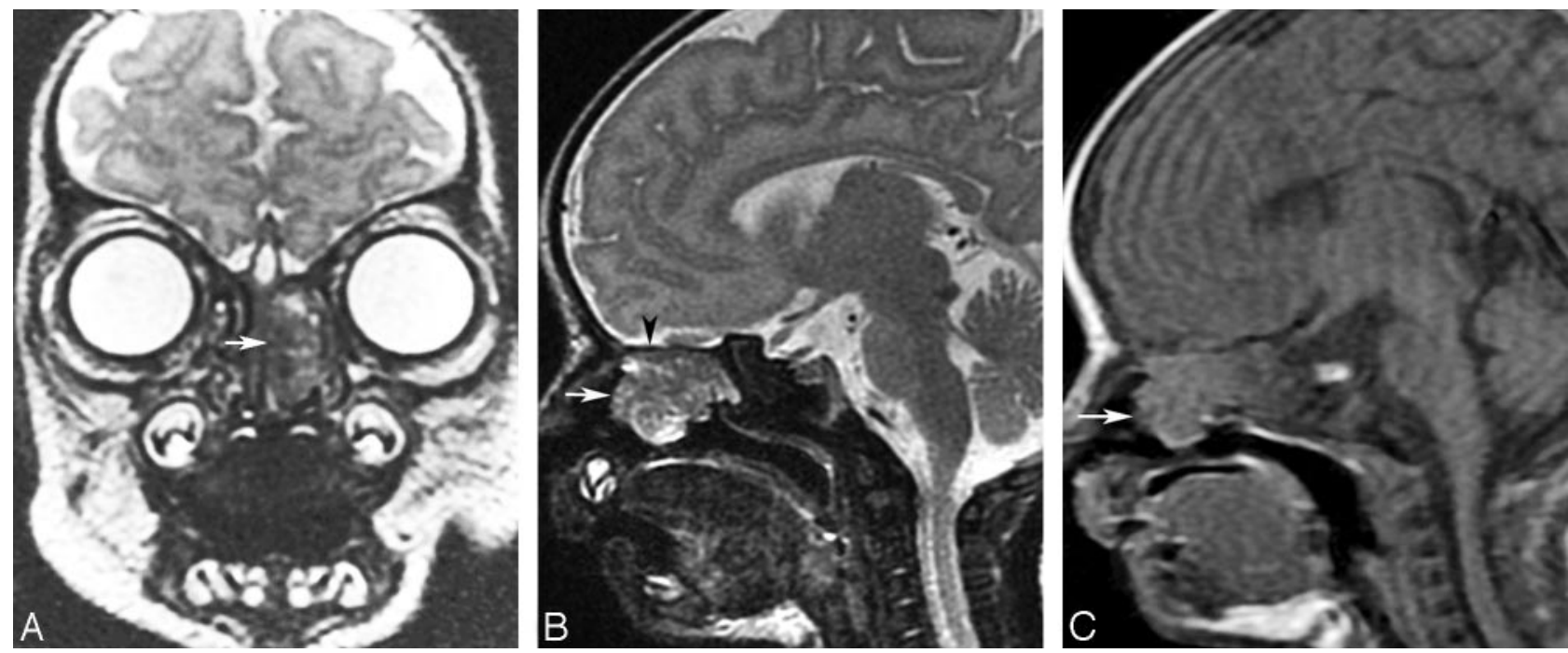

Fig 1. Coronal $(A)$ and sagittal $(B)$ T2-weighted and sagittal T1-weighted $(C)$ MR images show a well-circumscribed mass (white arrows) in the left nasal cavity and ethmoid labyrinth that is T1 isointense and heterogeneously T2 iso-hyperintense in relationship to brain parenchyma. The intact skull base is clearly visualized with no intracranial connection (black arrowhead).

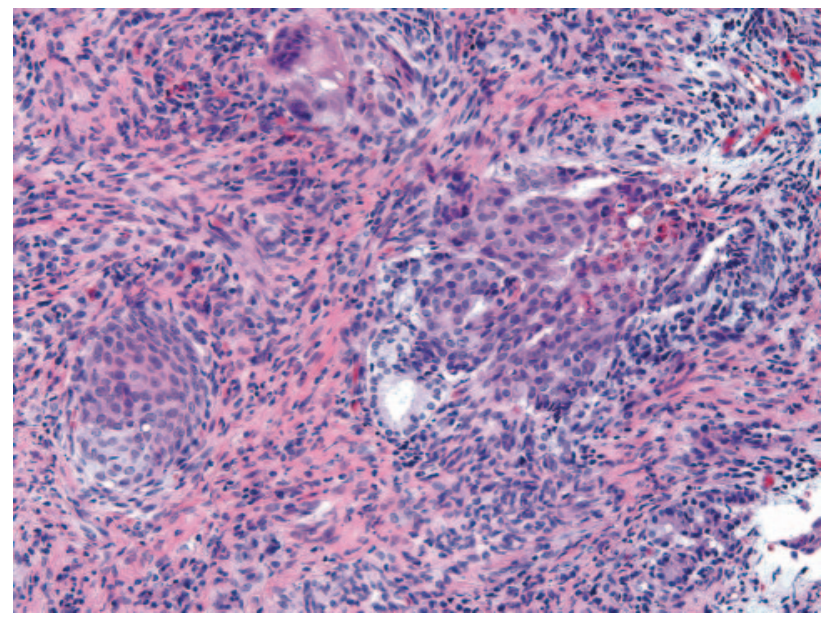

Fig 2. The pathologic features of SGAT: the biphasic nature of this hamartomatous lesion is demonstrated by the admixture of epithelial groups of ductlike and squamous structures and bland stromal cells (hematoxylin-eosin $\times 200$ ).

basis of the imaging appearance, the main consideration was a nasal glioma, a more common lesion that consists of disorganized, trapped brain tissue with no intracranial connection. As in the SGAT in our patient, nasal gliomas are typically T1 isointense and T2 iso-hyperintense to brain parenchyma. ${ }^{6}$

The causes of nasal or nasopharyngeal obstruction, unilateral or bilateral, in the neonate include developmental abnormalities such as nasal cavity or choanal stenosis or atresia. ${ }^{6}$ Masses in the nasal cavity and nasopharynx are rare in the neonate but are an important consideration in the setting of respiratory distress. The most common developmental masses are basal cephalocele, nasal glioma (neuroglial heterotopia), and nasolacrimal mucocele (dacryocystocele). Most neoplas- tic lesions involving the neonatal nasal and nasopharyngeal airway are benign such as dermoid, hamartoma, teratoma (epignathus), and hemangioma. Primary and secondary malignant neoplasms of this region are mainly seen in older infants and children and are extremely rare in the neonate. These lesions include neuroblastoma, Langerhans cell histiocytosis, chloroma, rhabdomyosarcoma, and lymphoma.

Congenital SGAT is usually not suggested as a possible cause in the setting of nasal obstruction from a mass in the neonatal period because it is uncommon. However, if this lesion is considered in the differential diagnosis on the basis of imaging studies, it is important to convey this to the referring physician because these lesions are attached by only a thin, delicate vascular pedicle that may be easily torn with gentle probing or nasal cannulation, resulting in dislodgement with possible unexpected complete airway obstruction.

\section{References}

1. Dehner LP, Valbuena L, Perez-Atayde A, et al. Salivary gland anlage tumor (“congenital pleomorphic adenoma"). A clinicopathologic, immunohistochemical and ultrastructural study of nine cases. Am J Surg Pathol 1994;18:25-36

2. Stillwater LB, Fee WE Jr. Squamous cell proliferative lesion of the nasopharynx in a newborn. Otolaryngol Head Neck Surg 1980;88:240-47

3. Har-El G, Zirkin HY, Tovi F, et al. Congenital pleomorphic adenoma of the nasopharynx (report of a case). J Laryngol Otol 1985;99:1281-87

4. Herrmann BW, Dehner LP, Lieu JE. Congenital salivary gland anlage tumor: a case series and review of the literature. Int $J$ of Pediatr Otorhinolaryngol 2005;69:149-56

5. Cohen EG, Yoder M, Thomas RM, et al. Congenital salivary gland anlage tumor of the nasopharynx. Pediatrics 2003;112:e66-e69

6. Valencia MP, Castillo M. Congenital and acquired lesions of the nasal septum: a practical guide for differential diagnosis. Radiographics 2008;28: 205-24 